\title{
Search for Neutrinoless Double Beta Decay with CUORE
}

\author{
Elena FERRI* on behalf of CUORE collaboration \\ Università Milano Bicocca - INFN Milano Bicocca, Italy \\ E-mail: Elena.ferriamib.infn.it
}

The Cryogenic Underground Observatory for Rare Events (CUORE) is an experiment to search for neutrinoless double beta decay $(\beta \beta 0 v)$ in ${ }^{130} \mathrm{Te}$ and other rare processes. The observation of $\beta \beta 0 \mathrm{v}$ would indicate that neutrinos are Majorana particles and would provide information about the absolute neutrino mass scale. CUORE is a bolometric detector composed of $988 \mathrm{TeO}_{2}$ crystals, with the total mass of about $741 \mathrm{~kg}$ of natural Tellurium. We will discuss the status of the CUORE experiment and present the most recent results from Cuoricino, the predecessor experiment operated in the Laboratori Nazionali del Gran Sasso (LNGS) in Italy.

The 2011 Europhysics Conference on High Energy Physics, EPS-HEP 2011,

July 21-27, 2011

Grenoble, Rhône-Alpes, France

\footnotetext{
* Speaker.
} 


\section{Cuoricino and CUORE}

Cuoricino and CUORE are calorimetric experiments searching for the Neutrinoless Double Beta Decay $(\beta \beta 0 v)$ of ${ }^{130} \mathrm{Te}$ with cryogenic detectors.

Cuoricino was an array of 62 crystals of $\mathrm{TeO}_{2}$ with a total mass of $40.7 \mathrm{~kg}$. It was running from February 2003 until June 2008 in Hall A of LNGS. The detectors were arranged in 13 planes tower like-structure: eleven modules were made of 4 crystals $5 \times 5 \times 5 \mathrm{~cm}^{3}$ each with a mass of $790 \mathrm{~g}$, and the other two modules of 9 crystals $3 \times 3 \times 6 \mathrm{~cm}^{3}$ with a mass of $330 \mathrm{~g}$. All the $\mathrm{TeO}_{2}$ crystals were characterized by a natural ${ }^{130} \mathrm{Te}$ isotopic abundance, except for 4 of the $330 \mathrm{~g}$ crystals. Two of them were enriched in ${ }^{130} \mathrm{Te}$ (i.a. $\sim 75 \%$ ) and the other two in ${ }^{128} \mathrm{Te}$ (i.a. $\sim 82 \%$ ). The final sum spectrum is obtained with the detectors operated in anticoincidence to reduce background contributions from $\mathrm{Th}$ and $\mathrm{U}$ chains on the crystals surfaces and from external gamma rays. The achieved background level in the $\beta \beta 0 \mathrm{v}$ energy region is $0.169 \pm 0.006 \mathrm{c} / \mathrm{KeV} / \mathrm{Kg} / \mathrm{y}$, which translates into a $90 \%$ C.L. lower limit on the half life of ${ }^{130} \mathrm{Te}$ of $\tau_{1 / 2}^{0 \nu} \geq 2.8 \times 10^{24} \mathrm{y}$. The corresponding upper limit for the effective Majorana mass is $m_{\beta \beta}<300-710 \mathrm{meV}$, the spread is due to uncertainties in nuclear matrix elements [四].

CUORE is an array of $988 \mathrm{TeO}_{2}$ crystals arranged in a cylindrical compact and granular structure of 19 towers. Each tower consists of 13 planes, 4 detectors $5 \times 5 \times 5 \mathrm{~cm}^{3}$ each $(750 \mathrm{~g})$. The active mass will be of $741 \mathrm{~kg}$, corresponding to $206 \mathrm{~kg}$ of ${ }^{130} \mathrm{Te}$. With a target background of 0.01 $\mathrm{c} / \mathrm{keV} / \mathrm{Kg} / \mathrm{y}$ and an energy resolution FWHM of about $5 \mathrm{keV}$ in the region of interest a 1 sigma sensitivity on $\tau_{1 / 2}^{0 v}$ of about $1.6 \times 10^{26}$ y can be achieved in five years $\left(m_{\beta \beta} \leq 41-95 \mathrm{meV}\right)$. The CUORE experiment is located in the Hall A of LNGS and it is due to start data taking in 2014. CUORE has the potentiality to probe the inverted hierarchy region of the neutrino mass pattern and its feasibility has been demonstrated by Cuoricino. A Monte-carlo projection of recent measurements onto CUORE background around $2.5 \mathrm{MeV}$ shows that almost all identified sources are controlled at the level of $10^{-3}$ counts $/ \mathrm{keV} / \mathrm{kg} / \mathrm{y}$. But the $\mathrm{U}$ and Th contaminations of the surfaces settle the current background contamination at around $0.02-0.04$ counts $/ \mathrm{keV} / \mathrm{kg} / \mathrm{y}$.

A final proof of the background achievements will be obtained by operating CUORE-0, the prototype tower to be installed in Cuoricino cryostat, which will take data until CUORE start. CUORE-0 represents a final test for several CUORE procedures like assembly, cleaning and gluing. The CUORE- 0 tower, composed by 52 detectors $750 \mathrm{~g}$ each, should start data taking before the end of the year. Moreover this measurement will be a self-consistent experiment by itself, soon overtaking Cuoricino sensitivity.

\section{Conclusion}

CUORE will be the first 1-ton experiment to search for the $\beta \beta 0 v$ of ${ }^{130} \mathrm{Te}$ using the bolometric technique. CUORE has the potentiality to probe the inverted hierarchy region of the neutrino mass pattern and its feasibility has been demonstrated by Cuoricino, a single tower running until 2008.

\section{References}

[1] E. Andreotti et al, ${ }^{130}$ Te Neutrinoless Double-Beta Decay with CUORICINO, Astropart. Phys. 34 (2011) 822 [arXiv:1012.3266]. 